01,13

\title{
Зарядовое состояние металлических наночастиц на проводящей подложке
}

\author{
(ㄱ Д.С. Ильющенков, В.М. Кожевин, С.А. Гуревич \\ Физико-технический институт им. А.Ф. Иофрфе РАН, \\ Санкт-Петербург, Россия \\ E-mail: dmitry@mail.ioffe.ru
}

Поступила в Редакцию 6 мая 2019 г.

В окончательной редакции 6 мая 2019 г.

Принята к публикации 14 мая 2019 г.

Работа посвящена анализу зарядового состояния неупорядоченной системы металлических наночастиц на проводящей подложке. Построена теоретическая модель, описывающая термостимулированные туннельные переходы электронов между частицами, а также обусловленные разностью работ выхода переходы электронов между частицами и подложкой. В модели учитывается взаимодействие зарядов ближайших частиц, а также взаимодействие зарядов частиц с зарядами изображения в подложке. Численное моделирование методом Монте-Карло для этой модели позволило получить картину распределения заряда в структурах с различной плотностью наночастиц в однослойном покрытии на подложке, при различных значениях диэлектрической проницаемости окружающей среды и различных величинах разницы работ выхода частиц и подложки. В качестве примера, приведены численные результаты для структур с наночастицами Ni и Pt на углеродной подложке, которые часто используются в качестве катализаторов химических процессов.

Ключевые слова: металлические наночастицы, зарядовое состояние, разница работ выхода.

DOI: 10.21883/FTT.2019.10.48241.471

\section{1. Введение}

Изучению явления переноса заряда в неупорядоченных системах металлических наночастиц посвящено значительное число работ [1-6]. Помимо фундаментального научного интереса, данное явление играет важную роль в ряде областей применения, прежде всего в катализе, поскольку структуры с металлическими наночастицами широко используются в качестве катализаторов химических процессов, при этом свойства катализатора в значительной мере определяются зарядовым состоянием наночастиц [7]. На практике система металлических наночастиц представляет собой покрытие, состоящее из частиц, расположенных случайным образом на поверхности подложки. Один из важных примеров подобной системы - структура с металлическими наночастицами, расположенными на поверхности диэлектрика с поверхностной плотностью менее одного монослоя. Если в такой системе средняя величина зазоров между частицами такова, что между ними могут происходить туннельные переходы электронов, то часть частиц в системе окажется заряженными. Детальная модель, описывающая зарядовое состояние таких структур с размером частиц порядка нескольких нанометров была представлена ранее в работе [8]. В этой работе была продемонстрирована важность роли флуктуаций заряда, обусловленных случайным распределением частиц на поверхности. Также было показано, что плотность заряда в системе возрастает с увеличением как диэлектрической проницаемости окружающей среды, так и плотности частиц на подложке ${ }^{1}$. Помимо зарядового взаимодействия между частицами, в ряде работ изучались также эффекты переноса заряда между металлическими наночастицами и непроводящими подложками, чаще всего из оксидов металлов [9-12]. В этом случае перенос заряда зависит от химического состава и структуры зон материала подложки, наличия примесей, величины работы выхода металла.

Иная картина имеет место в системе металлических наночастиц на проводящем носителе. В такой системе, помимо зарядового взаимодействия между частицами, важную роль играет перенос заряда между частицами и подложкой, который определяется разностью работ выхода [13]. В данном случае результат может сильно зависеть от качества поверхности подложки, свойств поверхности частиц, а также от свойств контакта частицаподложка. Если в области контакта с поверхностью подложки нет окислов или примесей и площадь контакта не слишком мала, то между частицей и подложкой происходит перераспределение плотности заряда, в результате чего частица как целое приобретает заряд, который не обязательно равен целому заряду электрона (дробный заряд) [14]. В противоположном случае выраженного туннельного контакта между частицей и подложкой электроны могут переходить с частицы на носитель или в обратном направлении, что может привести к изменению заряда частицы на величину, кратную

${ }^{1}$ В структурах большой плотности, с многослойным покрытием из плотноупакованных наночастиц на диэлектрической подложке, заряд делокализован в объеме покрытия, так что свойства покрытия становятся похожими на свойства дефектных металлов. 
заряду электрона [15]. По существу, разница этих двух режимов определяется величиной контактного сопротивления перехода частица-носитель. Как обсуждалось в $[16,17]$, если контактное сопротивление больше кванта сопротивления $h / q_{e}^{2}$, то происходит дискретный перенос заряда, кратного заряду электрона, если меньше имеет место дробное заряжение частиц. С помощью критерия величины контактного сопротивления можно также рассматривать перенос зарядов между соседними наночастицами в системе. Помимо свойств контакта частица-проводящий носитель, зарядовое состояние рассматриваемой системы может зависеть от наличия окислов (или примесей) как на поверхности подложки, так и на поверхности частиц [18]. Основная причина этого состоит в сильной зависимости работы выхода от состояния поверхности. Несмотря на достигнутое понимание основных факторов, влияющих на процессы переноса заряда в системе металлических наночастиц на проводнике, ряд вопросов, таких как особенности зарядового состояния, обусловленные дискретным характером заряжения частиц малого размера, роль беспорядка в системе частиц, остаются малоизученными.

В настоящей работе построена теоретическая модель, описывающая термостимулированные туннельные переходы электронов между частицами, а также обусловленные разностью работ выхода переходы между частицами и проводящей подложкой. Методом Монте-Карло проведено численное моделирование процесса формирования зарядового состояния системы в предположении, что основным фактором беспорядка в системе является случайное расположение частиц. Расчет вероятностей переходов проводится с учетом зарядовой энергии частиц, их взаимной поляризации и влияния зарядов изображения, наведенных вблизи поверхности носителя. Показано, что при малых размерах наночастиц, когда зарядовая энергия порядка разницы работ выхода, в состоянии теплового равновесия в системе не происходит выравнивания уровней Ферми частиц и носителя. Вместо этого возникают флуктуации зарядового состояния системы с энергиями заряженных частиц вблизи уровня Ферми. В качестве примера рассматривается зарядовое состояние наночастиц $\mathrm{Ni}$ и $\mathrm{Pd}$ на углероде, поскольку эти системы важны в катализе.

\section{2. Модель}

Модель, используемая в данной работе для описания зарядового состояния системы металлических наночастиц на проводящей подложке, является обобщением модели, представленной в [8]. Рассматриваются структуры, состоящие из одинаковых металлических наночастиц сферической формы, которые расположены случайным образом на поверхности проводящей подложки в виде одного, не полностью заполненного слоя. Степень заполнения слоя характеризуется параметром $\eta$, величина которого есть доля площади поверхности, занятая нано- частицами, $0<\eta<1$. Предполагается, что все частицы состоят из металлического ядра с радиусом $r$, покрытого тонкой непроводящей оболочкой (окислом) толщиной $d_{p}$. Таким образом, при случайной расстановке частиц в слое минимальное расстояние между центрами частиц равно $2 r+2 d_{p}$. Слой толщиной $d_{p}$ отделяет также ядро частицы от поверхности подложки. В модели предполагается, что толщина слоя $d_{p}$ не слишком мала, так что туннельные переходы электронов между частицами и между частицей и проводящей подложкой изменяют заряд частиц на величину, кратную заряду электрона.

При моделировании системы случайное расположение частиц в пределах слоя достигается путем включения процесса многократного соударения шаров (метод „бильярда“) [8]. Отметим, что в рассматриваемой системе случайное расположение частиц является единственным фактором беспорядка. Предположение об отсутствии разброса размеров частиц позволяет проанализировать влияние на свойства системы именно этого фактора беспорядка, который является характерным для большинства практических систем.

Рассмотрим вначале вероятность туннелирования заряда между наночастицами и подложкой. Эта вероятность описывается выражением [2]:

$$
\Gamma_{i \leftrightarrow s u b}=\alpha \exp \left(-2 \chi d_{p}\right) \cdot \frac{\Delta E_{i}^{ \pm}}{\exp \left(\Delta E_{i}^{ \pm} / k T\right)-1},
$$

где $\alpha$ - коэффициент, определяемый формой частиц, $\chi$ - туннельный фактор, $k$ - постоянная Больцмана, $T$ - температура, $\Delta E_{i}^{ \pm}$- энергия активации перехода электрона между подложкой и наночастицей $i$ (знак „+ ${ }^{\text {“ }}$ соответствует переходу электрона из подложки на наночастицу, а знак „-“ — переходу в обратном направлении). Энергия активации перехода $\Delta E_{i}^{ \pm}$зависит как от заряда самой наночастицы $i$, так и от заряда окружающих ее соседних наночастиц, обозначенных в дальнейшем индексом $j$, и определяется выражением:

$$
\Delta E_{i}^{ \pm}=\frac{q_{e}^{2}}{2} C_{i i}^{-1}\left(1 \pm 2 n_{i}\right) \pm q_{e}^{2} \sum_{i \neq j} C_{i i}^{-1} n_{j} \pm \Delta w,
$$

где $q_{e}-$ заряд электрона, $C_{i j}^{-1}$ - обратная матрица емкостей системы частиц, $n_{i}$ - исходное количество электронов на наночастице $i, n_{j}$ - количество электронов на окружающих наночастицах $j$, по которым в выражении (2) выполнено суммирование, $\Delta w$ - разница работ выхода подложки и наночастиц. Диагональный элемент матрицы $C_{i i}^{-1}$ - это обратная собственная емкость наночастицы $i$. Эта емкость определяет потенциал $p_{i}=q_{e} C_{i i}^{-1}$ данной наночастицы при помещении на нее пробного заряда, равного заряду электрона $q_{e}$, с учетом поляризации окружающих незаряженных наночастиц и проводящей подложки. Недиагональный элемент матрицы $C_{i j}^{-1}$ - это обратная взаимная емкость наночастиц $i$ и $j$. Взаимная емкость описывает потенциал $p_{i j}=q_{e} C_{i j}^{-1}$ 
нейтральной наночастицы $i$ при помещении пробного заряда $q_{e}$ на наночастицу $j$ также с учетом поляризации остальных окружающих незаряженных наночастиц и подложки. Матрица $C_{i j}^{-1}$ является симметричной, то есть обратные взаимные емкости наночастиц $i$ и $j$ совпадают: $C_{i j}^{-1}=C_{j i}^{-1}$. Выражение для энергии активации перехода (2) является обобщением соответствующего выражения, предложенного в работе [19], на случай перехода электрона между подложкой и наночастицей с учетом того, что материалы подложки и наночастиц могут иметь различающиеся работы выхода электрона.

Обратная матрица емкостей $C_{i j}^{-1}$ вычислялась при помощи пакета программ Comsol Multiphysics с использованием следующего подхода. В качестве расчетной области выбирается полусфера с центром, совпадающим с центром одной выбранной частицы. В качестве основания полусферы выступает поверхность подложки. Радиус полусферы выбирается таким образом, чтобы она вмещала достаточно большое количество частиц в окрестности выбранной частицы. Размер расчетной области выбирался таким образом, чтобы включать 24 частицы, ближайшие к выбранной. Считается, что поверхность расчетной области находится под нулевым потенциалом, что соответствует нулевому потенциалу на подложке. Объем частиц исключается из расчетной области, при этом поверхность частиц выступает в качестве границ расчетной области. Далее, на поверхности частиц в пределах расчетной области задается пробный потенциал и решается уравнение Лапласа $\Delta \varphi=0$, при этом потенциалы на поверхности наночастиц и нулевой потенциал на внешней поверхности расчетной области выступают в качестве граничных условий. Используя полученное решение, вычисляются интегралы электрического поля по поверхности каждой наночастицы, что дает значения заряда на всех наночастицах, которые соответствуют заданным потенциалам. Затем, при помощи метода оптимизации подбора параметров SNOPT [20], многократного решения уравнения Лапласа и интегрирования электрических полей, находится такой набор потенциалов наночастиц, который соответствует единичному заряду на центральной наночастице и нулевому заряду на остальных окружающих частицах. Полученный таким образом потенциал на центральной частице определяет ее собственную емкость $p_{i}=q_{e} C_{i i}^{-1}$, в то время, как потенциалы на окружающих частицах определяют взаимную емкость центральной частицы и окружающих частиц $p_{i j}=q_{e} C_{i j}^{-1}$. Для нахождения взаимной емкости выбранной частицы с частицами, находящимися вне расчетной области, использовалось приближенное значение $C_{i j} \sim r_{i j}$, где $r_{i j}-$ расстояние между центрами наночастиц. Для нахождения полной обратной матрицы емкостей $C_{i j}^{-1}$ указанная процедура применялась для каждой наночастицы в системе.

Важно отметить, что, хотя при вычислении собственных и взаимных емкостей наночастиц используется пробный электрический заряд, эти емкости не зависят от величины заряда и определяются исключительно геометрией системы: размером наночастиц и их взаимным расположением на проводящей подложке.

Описанный подход позволил полностью учесть эффект взаимной поляризации наночастиц и проводящей подложки. Это существенно повысило точность расчетов по сравнению с использованным в работе [8] дипольным приближением, которое плохо применимо для систем с относительно высокой плотностью наночастиц, в которых расстояние между наночастицами сопоставимо с их размерами.

Помимо туннельных переходов электронов между частицами и подложкой в рассматриваемой системе возможен также туннельный перенос заряда между соседними частицами. Вероятность перехода электрона между наночастицами $i$ и $j$ находится из выражения [8]:

$$
\Gamma_{i \rightarrow j}=\alpha \exp \left(-2 \chi d_{i j}\right) \cdot \frac{\Delta E_{i j}}{\exp \left(\Delta E_{i j} / k T\right)-1},
$$

где дополнительно использованы следующие обозначения: $d_{i j}-$ расстояние между поверхностями металлических ядер частиц $i$ и $j, \Delta E_{i j}$ - энергия активации прыжка $i \rightarrow j$, которая вычисляется согласно выражению, в которое входит обратная матрица емкостей $C_{i j}^{-1}$ :

$$
\Delta E_{i j}=\Delta E_{i}^{-1}+\Delta E_{j}^{+}-q_{e}^{2} C_{i j}^{-1} .
$$

Для вычисления энергий активации $\Delta E_{i}^{-}, \Delta E_{j}^{+}$используется выражение (2) без последнего слагаемого, связанного с разницей работ выхода.

Далее, процедура нахождения зарядового состояния системы состоит в следующем. С использованием полученной обратной матрицы емкостей $C_{i j}^{-1}$ и выражений (1)-(4), вычисляются вероятности всех возможных туннельных переходов электрона между наночастицами и проводящей подложкой и между соседними наночастицами. Затем, при помощи одноэлектронного метода Монте-Карло [21] с вероятностью, пропорциональной полученному распределению вероятностей переходов, осуществлялся выбор конкретного перехода, приводящего к изменению зарядового состояния наночастиц, между которыми произошел переход, либо зарядового состояния частицы при переходе электрона с частицы на подложку или обратно. После этого, для полученного зарядового состояния пересчитываются вероятности всех возможных переходов и выбирается новый переход, в результате которого реализуется новое зарядовое состояние. Процедура многократно повторяется до достижения квазистационарного зарядового состояния всей системы в целом. Важно подчеркнуть, что в квазистационарном состоянии величина суммарного заряда частиц перестает меняться на протяжении большого количества переходов в системе. Однако при этом происходят флуктуации зарядов как отдельных частиц, так и всей системы в целом.

Из выражений (1)-(4) следует, что в случае, когда разница работ выхода частиц и подложки сопоставима 
или превышает энергию активации межчастичных переходов, вероятность переноса заряда между частицами и подложкой доминирует над вероятностью межчастичных переходов. При этом переходы между частицами не оказывают заметного влияния на общее зарядовое состояние системы. Более того, влияние межчастичных переходов тем меньше, чем меньше плотность частиц на подложке. Действительно, при уменьшении плотности частиц вероятность межчастичных переходов резко падает вследствие увеличения зазоров между частицами, в то время как величина зазоров между частицами и подложкой остается постоянной и малой.

Отметим, что поскольку в данной модели для вычисления энергий активации переходов и обратной матрицы емкостей, используется подход, основанный на электростатике, модель имеет определенные ограничения. Полученные результаты будут корректными в случае, когда времена жизни зарядового состояния, обратно пропорциональные вероятностям переходов, существенно превышают время максвелловской релаксации в металлах.

\section{3. Результаты и обсуждение}

Разработанная модель использовалась для расчетов зарядового состояния систем с различной плотностью металлических наночастиц на проводящих подложках, в зависимости от разницы работ выхода частиц и подложки, при различных величинах диэлектрической проницаемости окружающей среды. Модельные структуры состояли из 1600 наночастиц, доля площади поверхности, занятая наночастицами, варьировалась в диапазоне $\eta=0.1-0.7$ (для сравнения, при плотной гексагональной упаковке доля занятой частицами площади составляет 0.907, при кубической - 0.785). Все расчеты выполнены для температуры $300 \mathrm{~K}$.

Ниже приведены результаты расчетов для структур с наночастицами $\mathrm{Ni}$ и $\mathrm{Pt}$, осажденными на углеродную подложку. Вследствие высокой химической активности наночастиц $\mathrm{Ni}$ и Pt такие структуры широко используются в качестве катализаторов, при этом, как отмечалось выше, свойства катализаторов в значительной мере определяются зарядовым состоянием системы наночастиц [7]. С точки зрения применимости модели важно, что технологии, используемые получения подобных структур обеспечивают контролируемую плотность частиц на подложке и достаточно малый разброс размеров частиц [22]. Рассматривая структуры, полученные в [22], в качестве примера, примем диаметр частиц $\mathrm{Ni}$ равным $2.5 \mathrm{~nm}$, частиц $\mathrm{Pt}-1.8 \mathrm{~nm}$. В расчетах данные наночастицы представлены в виде одинаковых шаров соответствующего размера, покрытых оболочкой окисла толщиной $0.2 \mathrm{~nm}$ (толщина монослоя окисла). Используя данные [23], разность работ выхода $\mathrm{Ni}$ и углерода примем равной $\Delta w=-0.25 \mathrm{eV}$, для структур с наночастицами Pt на углероде $\Delta w=+0.60 \mathrm{eV}$.

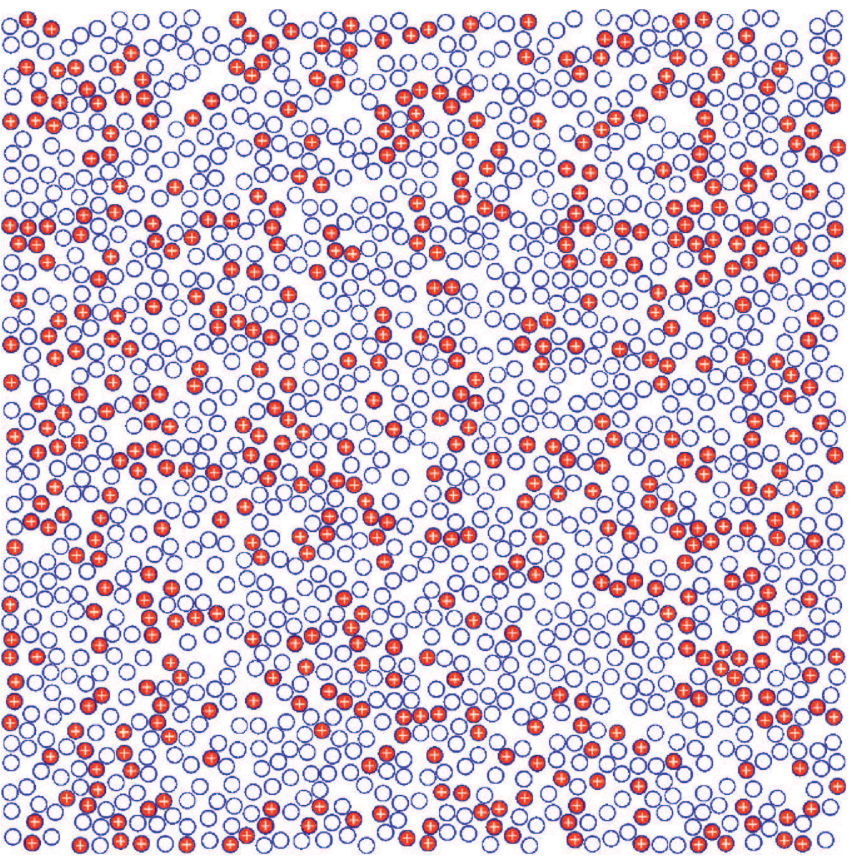

Рис. 1. Мгновенное распределение заряженных и нейтральных $2.5 \mathrm{~nm}$ наночастиц на подложке при разнице работ выхода $\Delta w=-0.25 \mathrm{eV}$, поверхностной плотности частиц $\eta=0.5$, диэлектрической приницаемости среды $\varepsilon=2$ и температуpe $300 \mathrm{~K}$.

На рис. 1 приведен результат расчета мгновенного распределения зарядов по ансамблю частиц Ni на углеродной подложке, находящихся в квазистационарном зарядовом состоянии. Как видно из рисунка, существенная доля частиц, около $30 \%$, однократно положительно заряжена, что соответствует переходу одного электрона из наночастицы на проводящую подложку. Доля заряженных частиц определяется величиной разницы работ выхода, $\Delta w=-0.25 \mathrm{eV}$, которая сопоставима с электростатической энергией электрона на наночастице размером $2.5 \mathrm{~nm}$.

На рис. 2 приведены гистограммы распределения по энергии наночастиц Ni с пробным однократным зарядом в окружении нейтральных частиц, построенные для разных значений плотности структур. При построении гистограмм последовательно каждая частица в системе рассматривалась как однократно заряженная в окружении нейтральных частиц, находилась ее энергия с учетом поляризационных эффектов и случайной расстановки окружения, после чего находилась доля частиц с определенной энергией. Как видно из рисунка, в распределении имеются характерные пики, которые соответствуют наночастицам, оказавшимся в различном окружении: без частиц в непосредственной близости, с одной, двумя или даже тремя ближайшими частицами. Данный результат является проявлением статистики случайной расстановки частиц. Как видно на рисунке, наибольшее значение электростатической энергии возникает в структурах ма- 


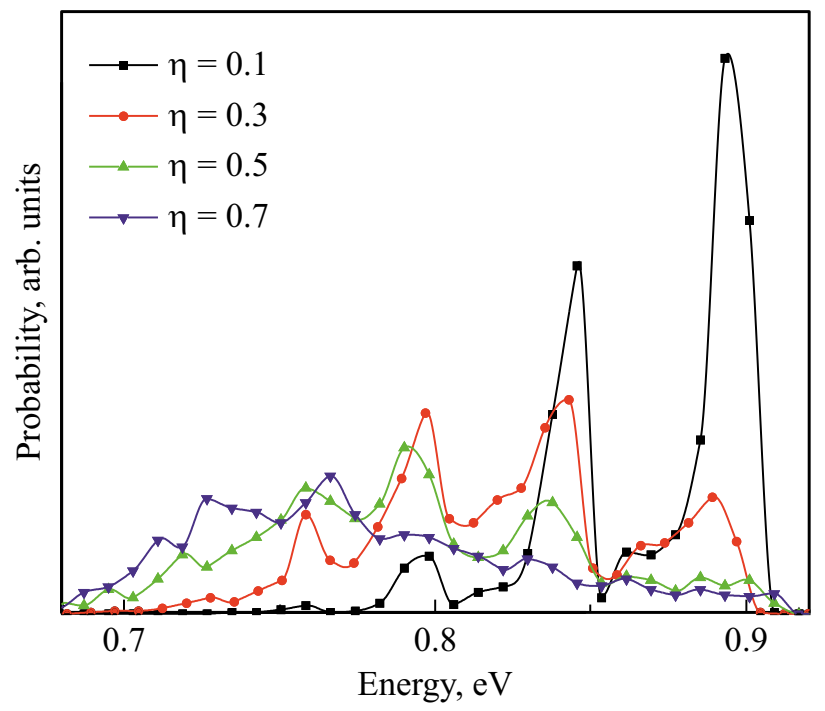

Рис. 2. Гистограмма распределения по энергии однократно заряженных наночастиц в окружении нейтральных частиц. Размер частиц $2.5 \mathrm{~nm}$, диэлектрическая приницаемость среды $\varepsilon=1$, температура $300 \mathrm{~K}$; расчеты при различной поверхностной плотности наночастиц $\eta=0.1-0.7$.

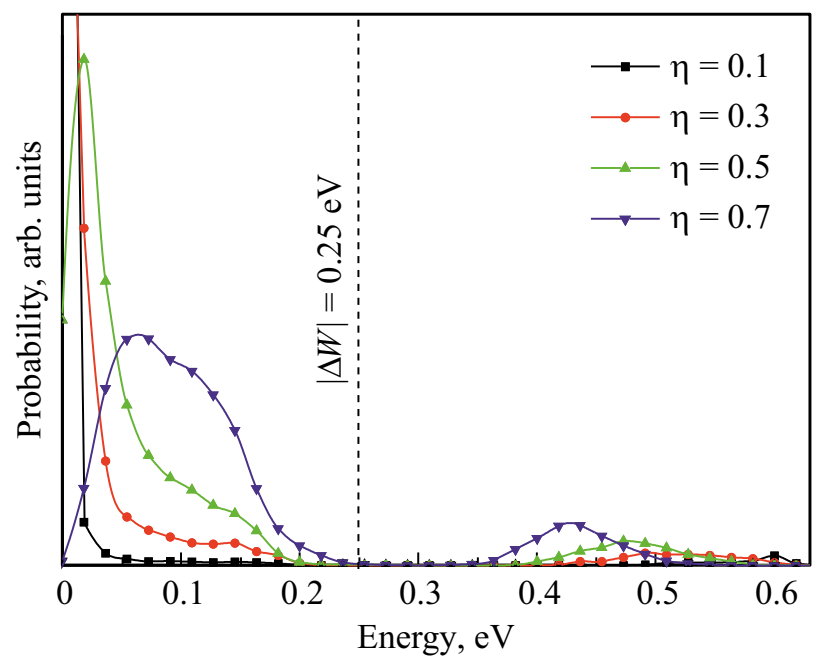

Рис. 3. Распределение по энергии заряженных и нейтральных наночастиц на проводящей подложке в квазистационарном состоянии системы. Размер частиц $2.5 \mathrm{~nm}$, углеродная подложка, разница работ выхода $\Delta w=-0.25 \mathrm{eV}$, диэлектрическая приницаемость среды $\varepsilon=1$, температура $300 \mathrm{~K}$, поверхностная плотность частиц $\eta=0.1-0.7$.

лой плотности, где имеется большое число уединенных частиц. При этом для частиц с ближайшими соседями эффекты поляризации соседних частиц приводят к понижению электростатической энергии.

Распределение по энергии наночастиц Ni на углеродной подложке в квазистационарном состоянии системы показано на рис. 3. Представленные данные были получены суммированием распределений по энергии всех частиц, заряженных и незаряженных, в различные моменты времени эволюции системы в квазистационарном состоянии. На рисунке вертикальная пунктирная линия соответствует положению уровня Ферми в углероде. Пики вероятности, расположенные слева от положения уровня Ферми в углероде, соответствуют нейтральным частицам, а расположенные справа однократно заряженным частицам. Ширина пиков в значительной мере определяется случайным характером расстановки частиц. Соотношение площадей под пиками на приведенных кривых отражает долю частиц с соответствующим зарядом. Как видно на рисунке, при увеличении поверхностной плотности доля заряженных частиц растет. Однако в случае $\varepsilon=1$, для которого проведен данный расчет, доля заряженных частиц мала и составляет менее $10 \%$ для всех значений плотности. Отметим, что наблюдаемое при увеличении плотности смещение пиков по энергии происходит как следствие усиления эффектов взаимной поляризации заряженных и незаряженных частиц. Таким образом, можно сделать вывод, что структура энергетических уровней наночастиц определяется не только геометрическими параметрами системы, но и долей заряженных частиц, которая в свою очередь зависит от разницы работ выхода частиц и подложки.

Важная особенность рассматриваемых систем, которую иллюстрирует рис. 3, состоит в том, что при достаточно большой разнице работ выхода и малом размере частиц положение их зарядовых уровней энергии значительно отличаться от положения уровня Ферми в подложке. Таким образом, выравнивание уровней Ферми, в том смысле, в котором оно имеет место при контакте двух объемных проводников, в системе наночастиц малого размера на проводящей подложке не достигается. Однако при усреднении по всему ансамблю частиц, если их достаточно много в системе, средневзвешенное положение уровня Ферми частиц должно совпадать с уровнем Ферми подложки.

На рис. 4 приведены данные о зарядовом состоянии ансамбля частиц $\mathrm{Ni}$ на углеродной подложке при различных значениях поверхностной плотности частиц и диэлектрической проницаемости среды. Как видно из рисунка, увеличение диэлектрической проницаемости приводит к росту числа заряженных частиц. В свою очередь, увеличение поверхностной плотности частиц приводит к росту поверхностной плотности заряда. При этом, удельный заряд частиц растет при увеличении поверхностной плотности частиц при $\varepsilon=1$ и уменьшается при $\varepsilon \geq 2$. Такое различие возникает из-за того, что при $\varepsilon=1$, число заряженных частиц в ансамбле мало, и основной вклад в изменение зарядовых уровней энергии при увеличении поверхностной плотности оказывают эффекты экранирования заряда окружающими нейтральными частицами. При больших значениях диэлектрической проницаемости и, соответственно, при увеличении доли заряженных частиц, преобладает увеличение зарядовой энергии, вызванное влиянием окружающих заряженных частиц. 


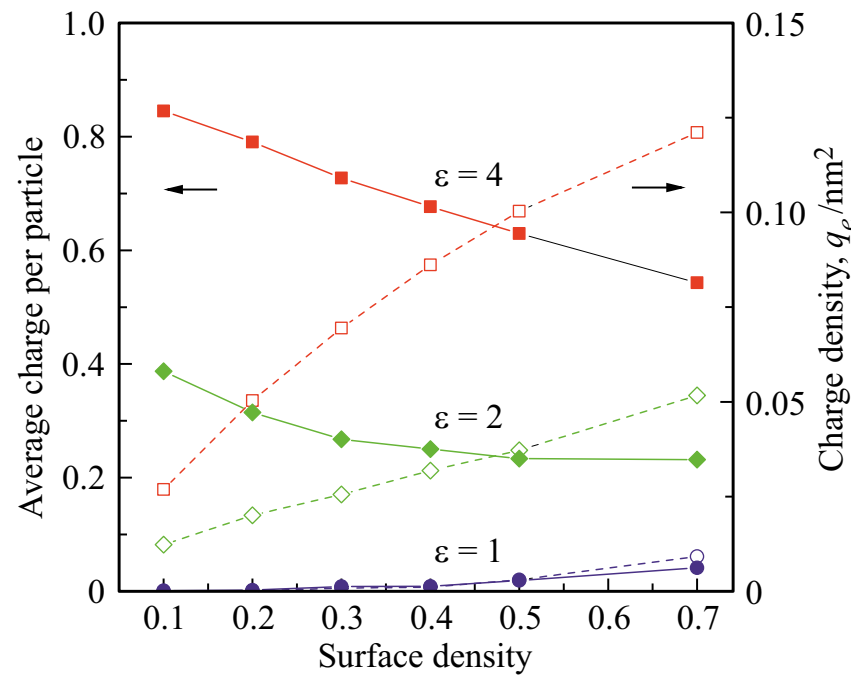

Рис. 4. Средний заряд, приходящийся на частицу, (сплошные кривые, левая ось) и поверхностная плотность заряда (пунктирные кривые, правая ось) для $2.5 \mathrm{~nm}$ наночастиц, при разнице работ выхода $\Delta w=-0.25 \mathrm{eV}$, при различных значениях диэлектрической проницаемости среды $\varepsilon=1$ в зависимости от поверхностной плотности наночастиц

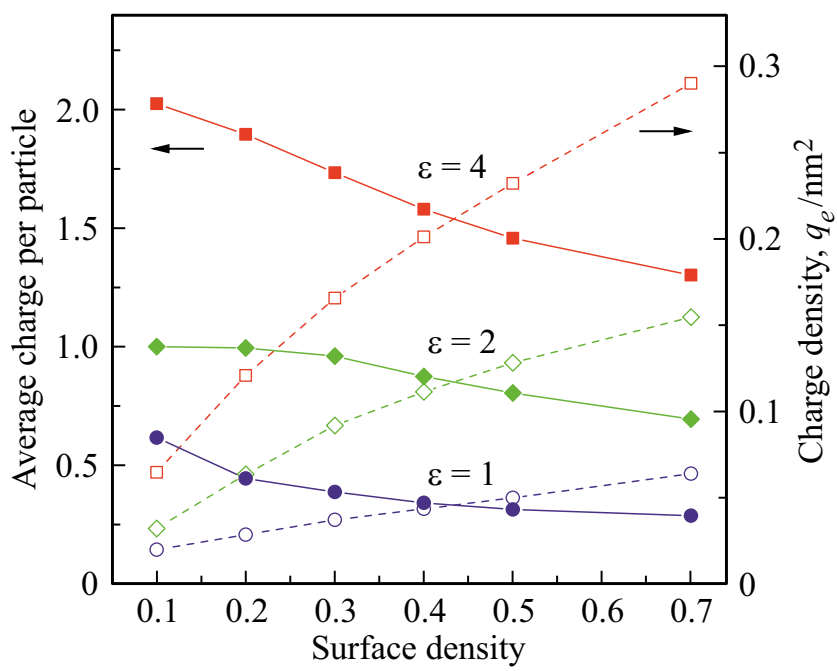

Pис. 5. Средний заряд, приходящийся на наночастицу, (сплошные кривые, левая ось) и поверхностная плотность заряда (пунктир, правая ось) для $1.8 \mathrm{~nm}$ наночастиц при разнице работ выхода $\Delta w=0.6 \mathrm{eV}$, при различных значениях диэлектрической проницаемости среды взависимости от поверхностной плотности наночастиц.

На рис. 5 показаны результаты расчетов зарядового состояния ансамбля наночастиц $\mathrm{Pt}$ на углеродной подложке. Как видно из рисунка, из-за большей разницы работ выхода $\Delta w=0.6 \mathrm{eV}$ доля заряженных частиц велика. Это приводит к преобладанию эффектов поляризации, вызванных окружающими заряженными частицами при всех значениях диэлектрической проницаемости, что вызывает рост зарядовой энергии и уменьшение среднего заряда, приходящегося на наночастицу, при увеличении поверхностной плотности частиц в слое.

Следует отметить, что во многих приложениях важной является не столько величина заряда частиц, сколько напряженность электрического поля, индуцированного этим зарядом вблизи поверхности частицы. Наиболее чувствительными к величине электрического поля являются химические каталитические процессы, протекающие на поверхности металлических наночастиц. Эти процессы многократно ускоряются, когда молекулы реагентов оказывается на поверхности в области электрических полей с напряженностью $E \geq 10^{7} \mathrm{~V} / \mathrm{cm}$ [24]. На рис. 6 показан результат расчета напряженности электрического поля в окрестности пары соприкасающихся наночастиц с диаметром $1.8 \mathrm{~nm}$. Картина распределения полей получена в результате решения уравнения Лапласа для случая, когда одна из частиц однократно заряжена, а вторая - нейтральная. Как видно из рисунка, в зазоре между заряженной частицей и подложкой величина электрического поля достигает $E \sim 2 \cdot 10^{7} \mathrm{~V} / \mathrm{cm}$. Такие величины напряженности поля достижимы только при достаточно малой величине собственной емкости частиц, что обеспечивается, в первую очередь, малостью размера частиц. Столь же сильное поле, с напряженностью $E \sim 10^{7} \mathrm{~V} / \mathrm{cm}$, имеется так же в зазоре между нейтральной частицей и подложкой. Это поле возникает благодаря поляризации, наведенной соседней заряженной частицей. Отметим, однако, что по мере увеличения зазора между заряженной и нейтральной наночастицами, напряженность электрического поля между ними быстро уменьшается: так при зазоре $1 \mathrm{~nm}$, электрическое поле спадает на порядок. Таким образом, в пленках с малой поверхностной плотностью сильные электрические поля локализованы между подложкой и заряженными частицами, в то время как в структурах большой плотности

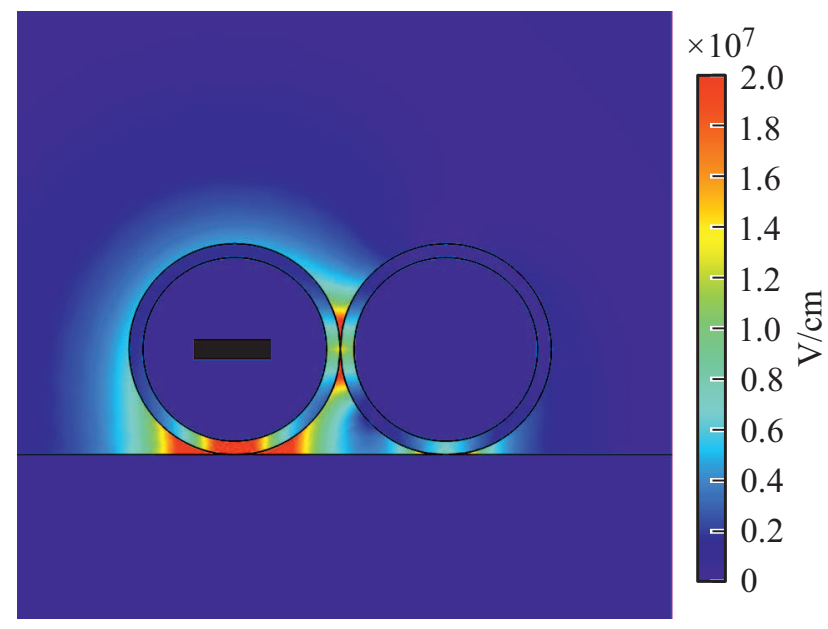

Рис. 6. Распределение напряженности электрического поля в окрестности пары однократно заряженной и нейтральной наночастицы, расположенных на проводящей подложке. Размер частиц $1.8 \mathrm{~nm}$, частицы покрыты оксидной оболочкой. 
области большой напряженности поля расположены как между соседними нейтральными и заряженными частицами, так и в зазорах между нейтральными частицами и поверхностью подложки.

\section{4. Заключение}

В работе построена теоретическая модель, описывающая спонтанное возникновение электрического заряда в гранулированных пленках, состоящих из металлических наночастиц, расположенных в случайном порядке в пределах одного слоя на проводящей подложке. В модели рассчитывается электростатическая энергия одноэлектронных переходов между наночастицами и подложкой и вычисляется частота таких переходов, что позволяет исследовать распределение зарядов в структуре. Показано, что основными факторами, влияющими на формирование зарядового состояния структур, являются плотность частиц в слое, диэлектрическая проницаемость среды и разница работ выхода материалов наночастиц и подложки.

Методом Монте-Карло проведено численное моделирование зарядового состояния структур для различных плотностей наночастиц в пленке и при различных значениях диэлектрической проницаемости среды. В результате расчётов получены значения квазистационарного уровня электрического заряда, возникающего в пленке в результате спонтанных одноэлектронных переходов между наночастицами и подложкой. Показано, что с увеличением плотности наночастиц происходит увеличение поверхностной плотности заряда в пленках, при этом поляризационные эффекты приводят к уменышению удельного заряда, приходящегося на наночастицу. Увеличение диэлектрической проницаемости среды вызывает понижение зарядовой энергии частиц и приводит к увеличению удельной плотности заряда. Показано, что в рассматриваемых структурах в зазорах между частицами и подложкой, а также в зазорах между частицами могут возникать сильные электрические поля с напряженностью до $10^{7} \mathrm{~V} / \mathrm{cm}$.

\section{Финансирование работы}

Работа выполнена в рамках темы госзадания ФТИ им. А.Ф. Иоффе.

\section{Конфликт интересов}

Авторы заявляют, что у них нет конфликта интересов.
[4] E. Cuevas, M. Ortuno, J. Ruiz. Phys. Rev. Lett. 71, 1871 (1993).

[5] Е.3. Мейлихов. ЖЭТФ 120, 712 (2001).

[6] A.A. Licalter. Physica A 291, 144 (2001).

[7] T.N. Rostovshchikova, E.S. Lokteva, E.V. Golubina, K.I. Maslakov, S.A. Gurevich, D.A. Yavsin, V.M. Kozhevin, Advanced Size-Selected Catalysts Prepared by Laser Electrodispersion, in Advanced Nanomaterials for Catalysis and Energy Synthesis, Characterization and Applications / Ed. Vladislav A. Sadykov. Elsevier, Amsterdam (2018), P. 61-94.

[8] Д.С. Ильющенков, В.М. Кожевин, С.А. Гуревич. ФТТ 57, 1670 (2015)

[9] G. Pacchioni. Phys. Chem. Chem. Phys. 15, 1737 (2013).

[10] Y. Lykhach, S.M. Kozlov, T. Skála, A. Tovt, V. Stetsovych, N. Tsud, F. Dvořák, V. Johánek, A. Neitzel, J. Mysliveček, S. Fabris, V. Matolín, K.M. Neyman, J. Libuda. Nature Mater 15, 284 (2016).

[11] Y. Peng, B. Lu, N. Wang, L. Li, Sh. Chen. Phys. Chem. Chem. Phys. 19, 9336 (2017).

[12] P. Frondelius, A. Hellman, K. Honkala, H. Häkkinen, H. Grönbeck. Phys. Rev. B 78, 085426 (2008).

[13] L. Giordano, G. Pacchioni. Acc. Chem. Res. 44, 1244 (2011).

[14] Y. Zhang, O. Pluchery, L. Caillard, A-F. Lamic-Humblot, S. Casale, Y.J. Chabal, M. Salmeron. Nano Lett. 15, 51 (2015).

[15] С.А. Гуревич, Д.С. Ильющенков, Д.А. Явсин, Н.В. Глебова, А.А. Нечитайлов, Н.К. Зеленина, А.А. Томасов. Электрохимия 53, 642 (2017).

[16] D.V. Averin, K.K Likharev, in Mesoscopic Phenomena in Solids / Eds B.L. Altshuler, P.A. Lee, R.A. Webb. Elsevier, Amsterdam (1991). 576 p.

[17] W. Zwerger, M. Scharpf. Z. Phys. B: Condens. Matter 85, 421 (1991).

[18] E. Palacios-Lidón, C.R. Henry, C. Barth. ACS Catal. 4, 1838 (2014).

[19] D.A. Zakheim, I.V. Rozhanski, S.A. Gurevich. Microel. Eng. 69, 646 (2003).

[20] Ph.E. Gill, W. Murray, M.A. Saunders. SIAM Rev. 47, N 1, 99 (2005).

[21] C. Jacoboni, L. Reggiani. Rev. Mod. Phys. 55, 645 (1983).

[22] В.М. Кожевин, Д.А. Явсин, Д.С. Ильющенков, Т.Н. Ростовщикова, Е.С. Локтева, С.А. Гуревич, Зарядовые эффекты и каталитические свойства систем металлических наночастиц. В кн.: Синтез, строение и свойства металл/полупроводник содержащих наноструктурированных композитов / Под ред. Л.И. Трахтенберга, М.Я. Мельникова, Техносфера, М. (2016). 622 с.

[23] В.С. Фоменко. Эмиссионные свойства материалов. Наук. думка, Киев (1981). 339 с.

[24] H.J. Kreuzer. Surf. Interface Anal. 36, 372 (2004).

Редактор К.В. Емцев

\section{Список литературы}

[1] P. Sheng, B. Abeles, Y. Arie. Phys. Rev. Lett. 31, 44 (1973).

[2] B. Abeles, P. Sheng, M.D. Coutts, Y. Arie. Adv. Phys. 24, 407 (1975).

[3] J. Klafter, P. Sheng. J. Phys. C 17, L93 (1984). 